\title{
Selected contributions, resulting from presentations at the workshop "Modeling Science-Understanding, Forecasting and Communicating the Science System," held in Amsterdam, October 6-9, 2009
}

\author{
Guest Editors \\ Katy Börner, Indiana University \\ Wolfgang Glänzel, Katholieke Universiteit Leuven \\ Andrea Scharnhorst, The Royal Netherlands Academy of Arts and Sciences \\ (Data Archives and Networked Services and e-Humanities Group) \\ Peter van den Besselaar, Vrije Universiteit Amsterdam
}

